\title{
Verhulst Model of Interval Grey Number Based on Information Decomposing and Model Combination
}

\author{
Bo Zeng, ${ }^{1}$ Chuan Li, ${ }^{2}$ Guo Chen, ${ }^{3}$ and Wang Zhang ${ }^{3}$ \\ ${ }^{1}$ Strategical Planning College, Chongqing Technology and Business University, Chongqing 400067, China \\ ${ }^{2}$ Engineering Laboratory for Detection \& Control and Integrated System, Chongqing Technology and Business University, \\ Chongqing 400067, China \\ ${ }^{3}$ School of Electrical and Information Engineering, The University of Sydney, Sydney, NSW 2006, Australia
}

Correspondence should be addressed to Guo Chen; guo.chen@sydney.edu.au

Received 5 November 2013; Accepted 7 December 2013

Academic Editor: Han H. Choi

Copyright (C) 2013 Bo Zeng et al. This is an open access article distributed under the Creative Commons Attribution License, which permits unrestricted use, distribution, and reproduction in any medium, provided the original work is properly cited.

Grey Verhulst models are often employed to simulate the development tendency with the characteristic of saturated process of $S$ curve. However, the uncertainty of interval grey numbers will be increased since the boundaries of interval grey number are extended by the Axiom of nondecreasing grey degree in the existing Verhulst modeling method. In this paper, the interval grey number is divided into two real number parts, that is, "white" and "grey" parts. Then the "white" and "grey" parts are simulated and forecasted by building the grey Verhulst model and DGM $(1,1)$ model, respectively. To some degree, this method resolves the issue of amplifying the range of interval grey number. Finally, an example is used to compare the simulation performance between the new model and the traditional model, and the results show that the new model is superior to the other model.

\section{Introduction}

The grey theory, which was founded by a famous Chinese scholar Deng [1] based on the idea of "Grey Box" in 1982, is a novel method employed to study the uncertain problems with small sample and poor information [2]. As one of the important constituents of grey theory, grey prediction models attract dramatic attentions and are always studied actively by many scholars. Accumulating generation of sequence is an important method in the process of building a grey prediction model, and one can potentially uncover a development tendency existing in the process of accumulating grey quantities so that the characteristics and laws of integration hidden in the chaotic original data can be sufficiently revealed [3]. Based on the properties of sequence accumulative generation, the grey exponent laws of sequence can be founded and excavated, and the expressions of simulation and prediction can be deduced.

GM $(1,1)$ model, first proposed by Professor Deng, is a classical grey prediction model, and currently it is of the most significant advantages in its influence, research findings, and extensive applications [4]. After 30 years development, grey prediction models have been expanded from original GM $(1,1)$ model to a great many of novel prediction categories, such as GM $(1, N), \mathrm{GM}(0, \mathrm{~N}), \mathrm{GM}(2,1)$, and DGM $(1,1)$ [5]. The prediction objects are extended to sequence predictions, interval predictions, grey disaster predictions, grey waveform predictions, system predictions, and so forth.

However, the modeling objects of conventional grey prediction model are sequences with the rules of exponent or approximate exponent. Hence the precision of grey prediction is inferior with the nonmonotone increasing (or decreasing) sequence [6]. It is important to note that not all the actual systems in the world meet the rules of approximate exponent growth, such as the process of ground subsidence: slow subsidence in beginning, then suddenly quick subsidence, and finally from quick subsidence to slow subsidence evenly sometimes it stops [7]. The variation tendency of the whole subsidence process presents the $S$ curve. The common probabilistic methods have the characteristic of large samples modeling, and the samples need to satisfy typical probability 
distribution. Otherwise the effect of simulation or prediction is inferior. Therefore, the conventional GM $(1,1)$ model or its derived models and the probabilistic methods cannot effectively simulate in the process of saturation state. In this context, the grey Verhulst model is built to solve such problems.

Grey Verhulst model [8] is a special grey prediction model which is developed to deal with the simulation for small sample data sequence with the characteristic of approximate single peak. This model is capable of simulating the time sequence data with the characteristic of saturated S curved. At present, the grey Verhulst model has been widely used in the fields of commodity economy life analysis [9], landslide prediction [10-12], biological breeding prediction [13], traffic accidents [14], energy demand [15], and so forth. In order to improve the simulation and prediction performance of grey Verhulst model, various researches have been done, including the optimization of initial and background values $[16,17]$ and the analysis of modeling mechanism $[18,19]$. Those studies have boosted the development and improvement of grey Verhulst model.

However, grey Verhulst model is only able to be constructed based on real number sequence according to the existing research findings. Though interval grey number sequence is more common in grey system theory, the Verhulst model is not capable of building on such sequence. Along with the development of science and technology, increasing the complexity in society, this trend leads to a larger amount of information in grey degree used to describe such systems. Under this context, the conventional grey Verhulst and its expanded models based on real sequence cannot meet the actual needs of systems modeling. Therefore, a modeling method of grey Verhulst model of interval grey number sequence is developed based on kernel and grey degree by Professor Yang [20]. Such method expands the modeling objects of grey Verhulst model from real number to interval grey number. It is of an important significance for enriching the model system of grey number prediction theory.

However, the simulation and prediction of the "kernel" of interval grey number are achieved by building the $\operatorname{DGM}(1,1)$ model of kernel sequence, and the grey degree of simulated or forecasted interval grey number is determined by Axiom of nondecreasing grey degree [21]. It is actually a simplified approximate measure, and the grey degree of the aim interval grey number is added; the value range of the simulated or forecasted interval grey number is enlarged to some extent; resulting in the increasing uncertainty of the future grey number.

In this paper, the interval grey number is divided into two real number parts, that is, "white" and "grey" parts. Then the "white" and "grey" parts are simulated and forecasted by building the Verhulst model and DGM $(1,1)$ model, respectively. To some degree, this method solves the issue of amplification in the value range of interval grey number caused by Axiom of nondecreasing grey degree and has a positive significance for developing and improving the model system of Verhulst model of interval grey number.

\section{Information Decomposing of Interval Grey Number}

Definition 1 (see [20]). Assume that an interval grey number $\otimes(k) \in\left[a_{k}, b_{k}\right], b_{k} \geq a_{k}, k=1,2, \ldots, \otimes(k)$ can be decomposed equivalently to the following form:

$$
\otimes(k)=a_{k}+c_{k} \mu,
$$

where

$$
c_{k}=b_{k}-a_{k}, \quad \mu \in[0,1] .
$$

Formula (1) is called the standard form of interval grey number $\otimes(k)$. the interval grey number expressed by corresponding standard form is called the standard interval grey number. In Formula (1), $a_{k}$ and $c_{k}$, respectively, are the white and grey part of the interval grey number $\otimes(k)$.

In formula (1), when $\mu=0, \otimes(k)=a_{k}+c_{k} \mu=a_{k}$; when $\mu=1, \otimes(k)=a_{k}+c_{k} \mu=b_{k}$; in both cases above, the interval grey number $\otimes(k)$ is all real numbers. When $\mu \in(0,1), \otimes(k)$ is an interval grey number of uncertainty value.

Definition 2 (see [20]). Assume that an interval grey number sequence $X_{\otimes}=(\otimes(1), \otimes(2), \ldots, \otimes(n))$, where $\otimes(k) \in\left[a_{k}, b_{k}\right]$ and $k=1,2, \ldots, n$; all the interval grey numbers in $X_{\otimes}$ are expressed in the standard form in formula (1). Then, all white parts, which are real numbers, constitute a sequence named white part sequence, denoted as $W$; similarly, all grey parts constitute the grey part sequence, denoted as $G$, as follows:

$$
X_{\otimes}=(\otimes(1), \otimes(2), \ldots, \otimes(n)) \Longleftrightarrow\left\{\begin{array}{l}
W=\left(a_{1}, a_{2}, \ldots, a_{n}\right) \\
G=\left(c_{1}, c_{2}, \ldots, c_{n}\right),
\end{array}\right.
$$

where

$$
\otimes(k) \in\left[a_{k}, b_{k}\right]=a_{k}+c_{k} \mu, \quad k=1,2, \ldots, n
$$

\section{Novel Verhulst Model}

\subsection{Verhulst Model Based on Real Part Sequence}

Definition 3 (see [4]). According to Definition 1, $W$ is the white part sequence of interval grey number sequence $X_{\otimes}$; let $W^{(1)}$ be the 1-AGO of $W$ and let $Z_{W}^{(1)}$ be the contiguous average value generating sequence, which are

$$
\begin{aligned}
W & =\left(a_{1}, a_{2}, \ldots, a_{n}\right) \\
\Longrightarrow W^{(1)} & =\left(a_{1}^{(1)}, a_{2}^{(1)}, \ldots, a_{n}^{(1)}\right), \\
W^{(1)} & =\left(a_{1}^{(1)}, a_{2}^{(1)}, \ldots, a_{n}^{(1)}\right) \\
\Longrightarrow Z_{W}^{(1)} & =\left(z_{w}^{(1)}(2), z_{w}^{(1)}(3), \ldots, z_{w}^{(1)}(n)\right),
\end{aligned}
$$

where

$$
a_{1}^{(k)}=\sum_{i=1}^{k} a_{i}, \quad z_{w}^{(1)}(k)=\frac{a_{k}^{(1)}+a_{k-1}^{(1)}}{2}, \quad k=2,3, \ldots, n .
$$


Then

$$
a_{k}+\varphi_{1} z_{w}^{(1)}(k)=\varphi_{2}\left(z_{w}^{(1)}(k)\right)^{2}
$$

is called the grey Verhulst model of the white part sequence of interval grey number.

Theorem 4 (see [4]). If one has sequences $W, W^{(1)}$, and $Z_{W}^{(1)}$ as described in Definitions 2 and 3 and if $\widehat{a}=[a, b]^{T}$ is a sequence parameters and

$$
Y=\left[\begin{array}{c}
a_{2} \\
a_{3} \\
\vdots \\
a_{n}
\end{array}\right], \quad B=\left[\begin{array}{cc}
-z_{w}^{(1)}(2) & \left(z_{w}^{(1)}(2)\right)^{2} \\
-z_{w}^{(1)}(3) & \left(z_{w}^{(1)}(3)\right)^{2} \\
\vdots & \vdots \\
-z_{w}^{(1)}(n) & \left(z_{w}^{(1)}(n)\right)^{2}
\end{array}\right],
$$

then

$$
\widehat{\varphi}=\left[\varphi_{1}, \varphi_{2}\right]^{T}=\left(B^{T} B\right)^{-1} B^{T} Y .
$$

According to formula (7) and Theorem 4, the time response expression of Verhulst model of white part sequence of interval grey number can be derived as follows:

$$
\widehat{a}_{k+1}^{(1)}=\frac{\varphi_{1} a_{1}^{(1)}}{\varphi_{2} a_{1}+\left(\varphi_{1}-\varphi_{2} a_{1}\right) e^{\varphi_{1} k}} .
$$

\subsection{DGM $(1,1)$ Model Based on Grey Part Sequence}

Definition 5. According to Definition 2, $G$ is the grey part sequence of interval grey number sequence $X_{\otimes}$; let $G^{(1)}$ be the 1-AGO of $G$ and let $Z_{G}^{(1)}$ be the contiguous average value generating sequence; that is,

$$
\begin{aligned}
G & =\left(c_{1}, c_{2}, \ldots, c_{n}\right) \\
\Longrightarrow G^{(1)} & =\left(c_{1}^{(1)}, c_{2}^{(1)}, \ldots, c_{n}^{(1)}\right) .
\end{aligned}
$$

Theorem 6. Assume that sequences $G, G^{(1)}$, and $Z_{G}^{(1)}$ are described in Definitions 2 and 5; if we have the sequence parameters $\widehat{\beta}=\left(\beta_{1}, \beta_{2}\right)^{T}$ of the DGM $(1,1)$ model and

$$
F=\left[\begin{array}{c}
c_{2}^{(1)} \\
c_{3}^{(1)} \\
\vdots \\
c_{n}^{(1)}
\end{array}\right], \quad E=\left[\begin{array}{cc}
c_{1}^{(1)} & 1 \\
c_{2}^{(1)} & 1 \\
\vdots & \vdots \\
c_{n-1}^{(1)} & 1
\end{array}\right]
$$

then

$$
\widehat{\beta}=\left(E^{T} E\right)^{-1} E^{T} F .
$$

According to literature [5] and Theorem 6, the time response expression of the $\operatorname{DGM}(1,1)$ model of the grey part sequence of interval grey number is derived as follows:

$$
\widehat{c}_{k+1}^{(1)}=\beta_{1}^{k} c_{1}+\frac{1-\beta_{1}^{k}}{1-\beta_{1}} \beta_{2} .
$$

According to literature [5], the final restore expression of the $\operatorname{DGM}(1,1)$ model of grey part sequence is as follows:

$$
\widehat{c}_{k+1}=\left(\beta_{1}-1\right)\left(c_{1}-\frac{\beta_{2}}{1-\beta_{1}}\right) \beta_{1}^{k} .
$$

3.3. Verhulst Model Based on Combination Models. The simulation and prediction are achieved by method of combining the white part sequence Verhulst model and grey part sequence-DGM $(1,1)$ model. On this basis, the upper and lower limit of interval grey number can then be simulated and forecasted.

In the practical situation, if the white part sequence of interval grey number is $S$ curved, the grey Verhulst and DGM $(1,1)$ model can be built by taking $W^{(1)}$ and $G^{(1)}$ as the raw data sequence. On the contrary, if the saturated $S$ curve can only be achieved by accumulating generation process on the white part sequence of interval grey number, then the grey combing models can only be built after the accumulating generation gets conducted. Hence, two difference models will be built according to the two circumstances mentioned above, as follows.

3.3.1. White Part Sequence of Interval Grey Number: Saturation $S$ Curve. Based on the above analysis, if the white part sequence of interval grey number has the $S$ curve, then grey Verhulst and DGM $(1,1)$ model can be built through taking $W^{(1)}$ and $G^{(1)}$ as the raw data sequence directly.

According to Definition $1, \widehat{c}_{k+1}=\widehat{b}_{k+1}-\widehat{a}_{k+1}$; then

$$
\widehat{b}_{k+1}^{(1)}=\widehat{c}_{k+1}+\widehat{a}_{k+1} .
$$

Combining formulas (10), (15), and (16), we get the following equations:

$$
\begin{gathered}
\widehat{a}_{k+1}^{(1)}=\frac{\varphi_{1} a_{1}^{(1)}}{\varphi_{2} a_{1}+\left(\varphi_{1}-\varphi_{2} a_{1}\right) e^{\varphi_{1} k},} \\
\widehat{c}_{k+1}^{(1)}=\beta_{1}^{k} c_{1}+\frac{1-\beta_{1}^{k}}{1-\beta_{1}} \beta_{2}, \\
\widehat{b}_{k+1}^{(1)}=\widehat{c}_{k+1}+\widehat{a}_{k+1} .
\end{gathered}
$$

By solving (17), the grey prediction model of the upper and lower limit of interval grey number $\widehat{\otimes}(k+1) \in\left[\widehat{a}_{k+1}^{(1)}, \widehat{b}_{k+1}^{(1)}\right]$ can be built, as follows,

$$
\begin{gathered}
\widehat{a}_{k+1}^{(1)}=\frac{\varphi_{1} a_{1}^{(1)}}{\varphi_{2} a_{1}+\left(\varphi_{1}-\varphi_{2} a_{1}\right) e^{\varphi_{1} k}}, \\
\widehat{b}_{k+1}^{(1)}=\beta_{1}^{k} c_{1}+\frac{1-\beta_{1}^{k}}{1-\beta_{1}} \beta_{2}+\frac{\varphi_{1} a_{1}^{(1)}}{\varphi_{2} a_{1}+\left(\varphi_{1}-\varphi_{2} a_{1}\right) e^{\varphi_{1} k}} .
\end{gathered}
$$

3.3.2. White Part Sequence of Interval Grey Number: Nonsaturation $S$ Curve. If the white part sequence of interval grey number is not saturation $S$ curve, firstly, the operation 
of accumulating generation needs to be applied to white part sequence $W$ and grey part sequence $G$. Following by building the grey Verhulst and DGM $(1,1)$ model based on $W^{(1)}$ and $G^{(1)}$, respectively. Finally, the simulation of white and grey part sequence is achieved by inverse accumulating generation.

For $\widehat{a}_{k+1}=\widehat{a}_{k+1}^{(1)}-\widehat{a}_{k}^{(1)}$, according to formula (10), $\widehat{a}_{k+1}$ can be derived as follows:

$$
\begin{aligned}
\widehat{a}_{k+1}= & \frac{\varphi_{1} a_{1}^{(1)}}{\varphi_{2} a_{1}+\left(\varphi_{1}-\varphi_{2} a_{1}\right) e^{\varphi_{1} k}} \\
& -\frac{\varphi_{1} a_{1}^{(1)}}{\varphi_{2} a_{1}+\left(\varphi_{1}-\varphi_{2} a_{1}\right) e^{\varphi_{1}(k-1)}} .
\end{aligned}
$$

According to Definition 1, $\widehat{c}_{k+1}=\widehat{b}_{k+1}-\widehat{a}_{k+1}$; then

$$
\begin{aligned}
\widehat{b}_{k+1} & =\widehat{a}_{k+1}+\widehat{c}_{k+1} \\
\Longrightarrow \widehat{b}_{k+1} & =\widehat{a}_{k+1}+\left(\beta_{1}-1\right)\left(c_{1}-\frac{\beta_{2}}{1-\beta_{1}}\right) \beta_{1}^{k} .
\end{aligned}
$$

Combining formula (19) and (20), we can get a formula group as follows:

$$
\begin{aligned}
\widehat{a}_{k+1}= & \frac{\varphi_{1} a_{1}^{(1)}}{\varphi_{2} a_{1}+\left(\varphi_{1}-\varphi_{2} a_{1}\right) e^{\varphi_{1} k}} \\
& -\frac{\varphi_{1} a_{1}^{(1)}}{\varphi_{2} a_{1}+\left(\varphi_{1}-\varphi_{2} a_{1}\right) e^{\varphi_{1}(k-1)}}, \\
\widehat{b}_{k+1}= & \left(\beta_{1}-1\right)\left(c_{1}-\frac{\beta_{2}}{1-\beta_{1}}\right) \beta_{1}^{k} \\
& +\frac{\varphi_{1} a_{1}^{(1)}}{\varphi_{2} a_{1}+\left(\varphi_{1}-\varphi_{2} a_{1}\right) e^{\varphi_{1} k}} \\
& -\frac{\varphi_{1} a_{1}^{(1)}}{\varphi_{2} a_{1}+\left(\varphi_{1}-\varphi_{2} a_{1}\right) e^{\varphi_{1}(k-1)}} .
\end{aligned}
$$

Formulas (18) and (21) are called the Verhulst model of interval grey number based on information decomposing and models combination.

\section{Comparison and Analysis of Models}

Exploiting groundwater is one of the main methods for providing people with living and industrial water in north China. However, overextraction of groundwater will lead to ground subsidence, which might cause disastrous consequences. In order to formulate the amount of groundwater mining scientifically and build the prediction and early warning system of ground subsidence, it is crucial to have the ability to forecast the future amount of ground subsidence according to historical monitoring data, so that the prediction data can be provided to support the research on the solutions of ground subsidence issues.

The process of ground subsidence is as follows: slow subsidence in beginning, then suddenly quick subsidence, and finally from quick subsidence to slow subsidence evenly sometimes it stops, and the variation tendency of the whole subsidence process presents the $\mathrm{S}$ curve. Hence, we plan on applying grey Verhulst model to forecast the amount of ground subsidence. Generally, the monitoring of ground subsidence amount for certain area is carried out in multiple continuous periods of time, and in each period the amount of ground subsidence will be monitored repetitively. Due to the monitoring errors and human factors, the collected monitoring data within the same area and period are not identical, neither are we able to identify the reliability of the data. At this time, in order to keep the data intact, an interval data will be defined according to the minimum and maximum values of the collected monitoring data. Assume that monitoring values of certain area are as shown in Table 1.

In this section, the Verhulst model of interval grey number based on information decomposing and models combination will be built according to data in Table 1 . Then we will compare and analyze its simulation errors with other model in literatures [20]. It is obvious that the data in Table 1 constitute an interval grey number sequence as follows:

$$
\begin{aligned}
X_{\otimes} & =(\otimes(1), \otimes(2), \otimes(3), \otimes(4), \otimes(5), \otimes(6)) \\
& =([6.4,9.3],[13.1,15.2],[19.1,21.3],[21.8,24.1],
\end{aligned}
$$$$
[21.6,23.8],[21.4,23.5]) \text {. }
$$

4.1. Modeling with the Novel Model Proposed in This Paper, Model 1 for Short. At first, it needs to transform the interval grey number sequence $X_{\otimes}$ into a real part and grey part sequence. According to formula (1), the real part sequence $W$ and grey part sequence $G$ are as follows:

$$
\begin{gathered}
W=(6.4,13.1,19.1,21.8,21.6,21.4), \\
G=(2.9,2.1,2.2,2.3,2.2,2.1) .
\end{gathered}
$$

The scatter point broken line figure of real part sequence $W$ and grey part sequence $G$ can be shown in Figure 1, as follows.

In Figure 1, it can be shown that the real part sequence is saturation $S$ curve and grey part sequence has the characteristic of approximate exponent laws. Hence, the grey Verhulst model of interval grey number sequence $X_{\otimes}$ can be built by applying formula (18).

4.1.1. Verhulst Model Based on Real Part Sequence. Computing the parameters of grey Verhulst model of the real part sequence $W$, according to Theorem 4 , matrix $B$ and $Y$ are as follows:

$$
B=\left[\begin{array}{cc}
-9.75 & (9.75)^{2} \\
-16.10 & (16.10)^{2} \\
-20.45 & (20.45)^{2} \\
-21.70 & (21.70)^{2} \\
-21.50 & (21.50)^{2}
\end{array}\right], \quad Y=\left[\begin{array}{l}
13.1 \\
19.1 \\
21.8 \\
21.6 \\
21.4
\end{array}\right]
$$

Then

$$
\widehat{\varphi}=\left[\varphi_{1}, \varphi_{2}\right]^{T}=\left(B^{T} B\right)^{-1} B^{T} Y=[-1.3316,-0.0610]^{T} .
$$


TABLE 1: Monitoring data of certain area within six continual periods.

\begin{tabular}{lcccccc}
\hline Monitoring period $p$ & $p=1$ & $p=2$ & $p=3$ & $p=4$ & $p=5$ & $p=6$ \\
\hline Data scope & {$[6.4,9.3]$} & {$[13.1,15.2]$} & {$[19.1,21.3]$} & {$[21.8,24.1]$} & {$[21.6,23.8]$} & {$[21.4,23.5]$} \\
\hline
\end{tabular}

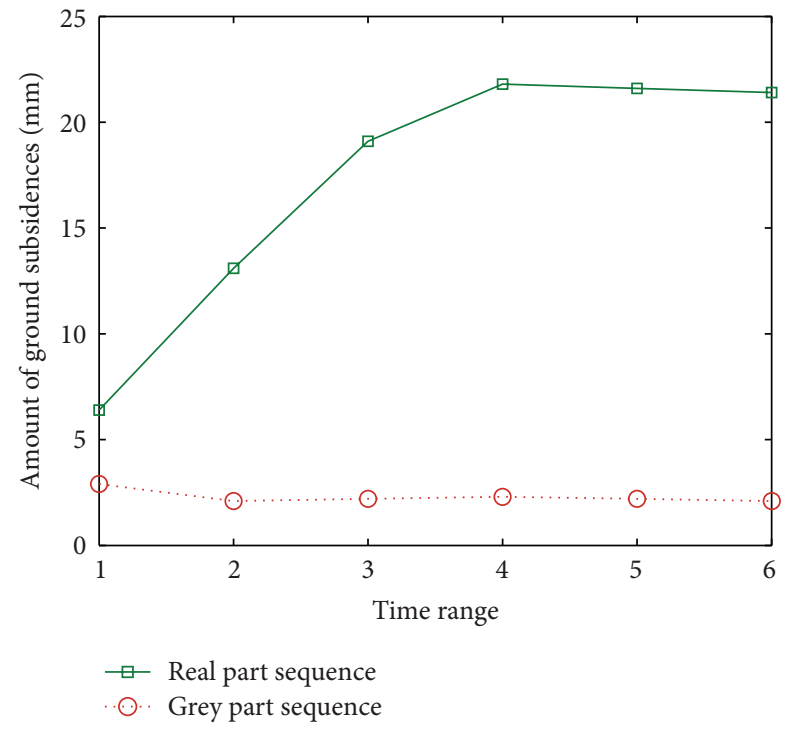

FIGURE 1: Scatter point broken line figure of real part sequence $W$ and grey part sequence $G$.

According to formula (10), the grey Verhulst model of the real part sequence $W$ of interval grey number sequence $X_{\otimes}$ is as follows:

$$
\begin{aligned}
\hat{a}_{k+1}^{(1)}= & \frac{\varphi_{1} a_{1}}{\varphi_{2} a_{1}+\left(\varphi_{1}-\varphi_{2} a_{1}\right) e^{\varphi_{1} k}} \\
= & (-1.3316 \times 6.4) \\
& \times(-0.0610 \times 6.4+[-1.3316-(-0.0610 \times 6.4)] \\
& \left.\quad \times e^{-1.3316 k}\right)^{-1} \\
\Longrightarrow \widehat{a}_{k+1}^{(1)}= & \frac{8.5222}{0.3904+0.9412 \times e^{-1.3316 k}} .
\end{aligned}
$$

4.1.2. DGM $(1,1)$ Model Based on Grey Part Sequence. Computing the parameters of DGM $(1,1)$ model of the grey part sequence $G$, according to Theorem 6, matrix $F$ and $E$ are as follows:

$$
F=\left[\begin{array}{c}
2.1 \\
2.2 \\
\vdots \\
2.1
\end{array}\right], \quad E=\left[\begin{array}{cc}
2.9 & 1 \\
2.1 & 1 \\
\vdots & \vdots \\
2.2 & 1
\end{array}\right]
$$

then

$$
\widehat{\beta}=\left(\beta_{1}, \beta_{2}\right)^{T}=\left(E^{T} E\right)^{-1} E^{T} F=(-0.1359,2.4981)^{T} .
$$

According to formula (14),

$$
\begin{aligned}
\widehat{c}_{k+1}^{(1)} & =\beta_{1}^{k} c_{1}+\frac{1-\beta_{1}^{k}}{1-\beta_{1}} \beta_{2}=c_{1} \beta_{1}^{k}+\frac{\beta_{2}}{1-\beta_{1}}\left(1-\beta_{1}^{k}\right) \\
\Longrightarrow & \widehat{c}_{k+1}^{(1)}=c_{1} \beta_{1}^{k}+\frac{\beta_{2}}{1-\beta_{1}}-\frac{\beta_{2}}{1-\beta_{1}} \beta_{1}^{k} \\
\Longrightarrow \widehat{c}_{k+1}^{(1)} & =\left(c_{1}-\frac{\beta_{2}}{1-\beta_{1}}\right) \beta_{1}^{k}+\frac{\beta_{2}}{1-\beta_{1}} .
\end{aligned}
$$

Then

$$
\begin{aligned}
\widehat{c}_{k+1}^{(1)} & =\left(2.9-\frac{2.4981}{1+0.1359}\right)(-0.1359)^{k}+\frac{2.4981}{1+0.1359} \\
\Longrightarrow \widehat{c}_{k+1}^{(1)} & =0.7007 \times(-0.1359)^{k}+2.1992 .
\end{aligned}
$$

4.1.3. Grey Verhulst Prediction Model of Amount of Ground Subsidence. According to formulas (18), the Verhulst prediction model of ground subsidence amount is derived as follows:

$$
\begin{aligned}
\widehat{a}_{k+1}^{(1)}= & \frac{\varphi_{1} a_{1}^{(1)}}{\varphi_{2} a_{1}+\left(\varphi_{1}-\varphi_{2} a_{1}\right) e^{\varphi_{1} k}}, \\
\widehat{b}_{k+1}^{(1)}= & \beta_{1}^{k} c_{1}+\frac{1-\beta_{1}^{k}}{1-\beta_{1}} \beta_{2}+\frac{\varphi_{1} a_{1}^{(1)}}{\varphi_{2} a_{1}+\left(\varphi_{1}-\varphi_{2} a_{1}\right) e^{\varphi_{1} k}} \\
\Longrightarrow \widehat{a}_{k+1}^{(1)}= & \frac{8.5222}{0.3904+0.9412 \times e^{-1.3316 k}}, \\
\widehat{b}_{k+1}^{(1)}= & 0.7007 \times(-0.1359)^{k} \\
& +\frac{8.5222}{0.3904+0.9412 \times e^{-1.3316 k}}+2.1992 .
\end{aligned}
$$

4.2. Modeling with the Model in Literature [20], Model 2 for Short. In literature [20], the information scope of interval grey number of prediction and simulation is determined by the axiom of nondecreasing grey degree, and the kernel of interval grey number is forecasted by grey Verhulst model. On the basis, the grey Verhulst model of interval grey number is derived.

4.2.1. Definition of Information. According to formula (1), it can be known that $c_{k}=b_{k}-a_{k}$ and $c_{k}$ which is the grey part of interval grey number is just the information scope that according to the axiom of nondecreasing grey degree, the maximum value in grey part sequence can be used to forecast 
TABLE 2: Simulation values and errors of the novel model.

\begin{tabular}{|c|c|c|c|c|c|c|c|c|}
\hline \multirow{2}{*}{ No } & \multicolumn{8}{|c|}{ Items } \\
\hline & $a_{p}$ & $\widehat{a}_{p}$ & $\varepsilon_{a}(p)$ & $\Delta_{a}(p)$ & $b_{p}$ & $\widehat{b}_{p}$ & $\varepsilon_{b}(p)$ & $\Delta_{b}(p)$ \\
\hline$p=2$ & 13.1 & 13.3 & -0.2 & $1.527 \%$ & 15.2 & 15.4 & -0.2 & $1.316 \%$ \\
\hline$p=3$ & 19.1 & 18.7 & 0.4 & $2.094 \%$ & 21.3 & 20.9 & -0.6 & $2.817 \%$ \\
\hline$p=4$ & 21.8 & 20.9 & 0.9 & $4.128 \%$ & 24.1 & 23.1 & 1.0 & $4.149 \%$ \\
\hline$p=5$ & 21.6 & 21.6 & 0.0 & $0.000 \%$ & 23.8 & 23.8 & 0.0 & $0.000 \%$ \\
\hline$p=6$ & 21.4 & 21.8 & -0.4 & $1.869 \%$ & 23.5 & 24.0 & -0.5 & $2.128 \%$ \\
\hline
\end{tabular}

or simulate the information scope of interval grey number; that is,

$$
\begin{aligned}
c & =\max \left\{c_{1}, c_{2}, \ldots, c_{n}\right\} \\
& =\max \{2.9,2.1,2.2,2.3,2.2,2.1\}=2.9
\end{aligned}
$$

4.2.2. Grey Verhulst Model of Kernel Sequence. Computing kernel sequence $X_{\widetilde{\otimes}}=(\widetilde{\otimes}(1), \widetilde{\otimes}(2), \widetilde{\otimes}(3), \widetilde{\otimes}(4), \widetilde{\otimes}(5), \widetilde{\otimes}(6))$ of interval grey number sequence $X_{\otimes}$, according to the computing method of kernel in literature [22], we can get

$$
\begin{aligned}
X_{\otimes} & =(\otimes(1), \otimes(2), \otimes(3), \otimes(4), \otimes(5), \otimes(6)) \\
\Longrightarrow X_{\tilde{\otimes}} & =(\widetilde{\otimes}(1), \widetilde{\otimes}(2), \widetilde{\otimes}(3), \widetilde{\otimes}(4), \widetilde{\otimes}(5), \widetilde{\otimes}(6)) \\
& =(7.85,14.15,20.20,22.95,22.70,22.45) .
\end{aligned}
$$

Building the Verhulst model of sequence $X_{\tilde{\otimes}}$, and its parameter $\widehat{a}=[a, b]^{T}=[-1.2248,-0.0532]^{T}$, then the Verhulst model of kernel sequence is as follows:

$$
\begin{aligned}
\widehat{\widetilde{\otimes}}^{(1)}(k+1)= & \frac{\widehat{a}_{k+1}+\widehat{b}_{k+1}}{2}=\frac{a \widetilde{\otimes}(1)}{b \widetilde{\otimes}(1)+(a-b \widetilde{\otimes}(1)) e^{a k}} \\
\Longrightarrow \widehat{\widetilde{\otimes}}^{(1)}(k+1)= & \frac{\widehat{a}_{k+1}+\widehat{b}_{k+1}}{2}=(-1.2248 \times 7.85) \\
& \times(-0.0532 \times 7.85 \\
& +(-1.2248+0.0532 \times 7.85) \\
& \left.\times e^{-1.2248 k}\right)^{-1} .
\end{aligned}
$$

4.2.3. Grey Verhulst Model of Interval Grey Number Sequence. Consider

$$
\begin{aligned}
\frac{\widehat{a}_{k+1}+\widehat{b}_{k+1}}{2} & =\widehat{\widetilde{\otimes}}^{(1)}(k+1), \\
c & =\widehat{b}_{k+1}-\widehat{a}_{k+1} \\
\Longrightarrow \widehat{a}_{k+1} & =\frac{a \widetilde{\otimes}(1)}{b \widetilde{\otimes}(1)+(a-b \widetilde{\otimes}(1)) e^{a k}}-\frac{c}{2}, \\
\widehat{b}_{k+1} & =\frac{a \widetilde{\otimes}(1)}{b \widetilde{\otimes}(1)+(a-b \widetilde{\otimes}(1)) e^{a k}}+\frac{c}{2}
\end{aligned}
$$

$$
\begin{aligned}
\Longrightarrow \widehat{a}_{k+1}=( & -1.2248 \times 7.85) \\
\times & (-0.0532 \times 7.85 \\
& +(-1.2248+0.0532 \times 7.85) \\
& \left.\times e^{-1.2248 k}\right)^{-1}-\frac{2.9}{2} \\
\widehat{b}_{k+1}= & (-1.2248 \times 7.85) \\
& \times(-0.0532 \times 7.85 \\
& +(-1.2248+0.0532 \times 7.85) \\
\widehat{a}_{k+1}= & \frac{\left.\times e^{-1.2248 k}\right)^{-1}+\frac{2.9}{2}}{-0.4176-0.8072 \times e^{-1.2248 k}}-1.45, \\
\widehat{b}_{k+1}= & \frac{-9.6147}{-0.4176-0.8072 \times e^{-1.2248 k}}+1.45 .
\end{aligned}
$$

4.3. Errors Comparisons and Analysis of the above Two Grey Verhulst Models of Interval Grey Number

Definition 7. Assume that an interval grey number sequence $X(\otimes)=\left(\otimes\left(t_{1}\right), \otimes\left(t_{2}\right), \ldots, \otimes\left(t_{n}\right)\right)$, and $\otimes\left(t_{p}\right) \in\left[a_{p}, b_{p}\right](k=$ $1,2, \ldots, n)$, an interval grey number prediction model of sequence $X(\otimes)$ is built with a modeling method $\aleph$, and the simulation sequence is $X(\widehat{\otimes})=\left(\widehat{\otimes}\left(t_{1}\right), \widehat{\otimes}\left(t_{2}\right), \ldots, \widehat{\otimes}\left(t_{n}\right)\right)$, and $\widehat{\otimes}\left(t_{p}\right) \in\left[\widehat{a}_{p}, \widehat{b}_{p}\right]$; then

$\left(1^{\circ}\right) \varepsilon_{a}(p)=a_{p}-\widehat{a}_{p}$ is the residual error of the upper limit $a_{p}$

$\left(2^{\circ}\right) \Delta_{a}(p)=\left|\varepsilon_{a}(p)\right| / a_{p}$ is the simulation relative error of the upper limit $a_{p}$;

$\left(3^{\circ}\right) \Delta_{a}=(1 /(n-1)) \sum_{p=2}^{n} \Delta_{a}(p)$ is the average simulation relative error of the upper limit sequence.

Similarly,

$\left(4^{\circ}\right) \varepsilon_{b}(p)=b_{p}-\hat{b}_{p}$ is the residual error of the lower limit $b_{p}$

$\left(5^{\circ}\right) \Delta_{b}(p)=\left|\varepsilon_{b}(p)\right| / b_{p}$ is the simulation relative error of the lower limit $b_{p}$; 
TABLE 3: Simulation values and errors of the model in literature [20].

\begin{tabular}{|c|c|c|c|c|c|c|c|c|}
\hline \multirow{2}{*}{ Number } & \multicolumn{8}{|c|}{ Items } \\
\hline & $a_{p}$ & $\widehat{a}_{p}$ & $\varepsilon_{a}(p)$ & $\Delta_{a}(p)$ & $b_{p}$ & $\widehat{b}_{p}$ & $\varepsilon_{b}(p)$ & $\Delta_{b}(p)$ \\
\hline$p=2$ & 13.1 & 13.2 & -0.1 & $0.763 \%$ & 15.2 & 16.1 & -0.9 & $5.921 \%$ \\
\hline$p=3$ & 19.1 & 18.3 & 0.8 & $4.189 \%$ & 21.3 & 21.2 & 0.1 & $0.469 \%$ \\
\hline$p=4$ & 21.8 & 20.5 & 1.3 & $5.963 \%$ & 24.1 & 23.4 & 0.7 & $2.905 \%$ \\
\hline$p=5$ & 21.6 & 21.2 & 0.4 & $1.852 \%$ & 23.8 & 24.1 & -0.3 & $1.261 \%$ \\
\hline$p=6$ & 21.4 & 21.5 & -0.1 & $0.467 \%$ & 23.5 & 24.4 & -0.9 & $3.830 \%$ \\
\hline
\end{tabular}

TABLE 4: Comparison of simulation errors with different models.

\begin{tabular}{lcc}
\hline Models & Model 1 & Model 2 \\
\hline Average simulation relative errors & $\Delta_{a}=1.924 \%, \Delta_{b}=2.082 \%$ & $\Delta_{a}=2.647 \%, \Delta_{b}=2.877 \%$ \\
Synthesis average simulation relative error & $\Delta_{1}=\frac{\Delta_{a}+\Delta_{b}}{2}=2.002 \%$ & $\Delta_{2}=\frac{\Delta_{a}+\Delta_{b}}{2}=2.762 \%$ \\
\hline
\end{tabular}

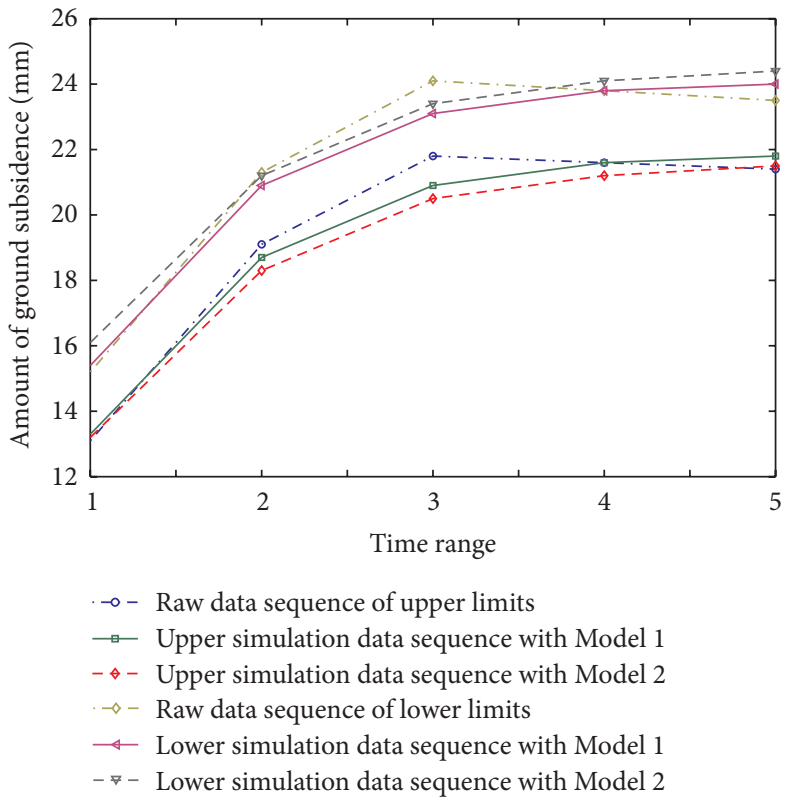

FIGURE 2: Comparison of simulation data with different models.

$\left(6^{\circ}\right) \Delta_{b}=(1 /(n-1)) \sum_{p=2}^{n} \Delta_{b}(p)$ is the average simulation relative error of the lower limit sequence.

Then $\Delta_{\mathcal{N}}=\left(\left(\Delta_{a}+\Delta_{b}\right) / 2\right) \times 100 \%$ is the synthesis average simulation relative error of sequence $X(\otimes)$ based on the modeling method $\aleph$.

According to Definition 7, the simulation errors of the above two models are shown from Table 2 to Table 3, respectively.

The comparison of simulation and raw data between grey Verhulst model proposed in this paper and model in literature [20] can be shown in Figure 2.

The comparison of simulation errors with different models is shown in Table 4, as follows.

It is easily to see from Figure 2 and Table 4 that the synthesis average simulation relative error of the novel model based on information decomposing and model combination is superior to the other model in literature [20].

\section{Conclusions and Future Work}

Grey Verhulst models are often employed to simulate the development tendency with the charactersitic of saturated $S$ curve process. However, the boundaries of interval grey number are extended through axiom of nondecreasing grey degree in the existing Verhulst modeling method. As a result, the uncertainty of the aim interval grey number will be added.

In order to solve the problem of prediction uncertainty amplification, the interval grey number is decomposed two real number parts, that is, "white" and "grey" part. Then the "white" and "grey" parts are simulated and forecasted by building the Verhulst model and DGM $(1,1)$ model, respectively. To some degree, this method solves the issue of amplification in the value range of interval grey number caused by axiom of nondecreasing grey degree and has a positive significance for developing and improving the model system of Verhulst model of interval grey number.

Apart from the advantages of our proposed model, there is a limitation in the application range of the proposed model. When the white part sequence of interval grey number has the $\mathrm{S}$ curve, the grey Verhulst model is directly built by taking $W^{(1)}$ as the raw data sequence, where the process of accumulative generation is omitted. Moreover, the $\operatorname{DGM}(1,1)$ model is assumed suitable to be built by the grey part sequence without accumulative generation process. Consequently, this simplified process might limit the range of application.

As for the next stage of research, we are going to extend the applicability of the Verhulst model of interval grey number by constructing and optimizing the model, based on the variation trends and data features of "white and grey" part sequence.

\section{Conflict of Interests}

The authors declare that there is no conflict of interests regarding the publication of this paper. 


\section{Acknowledgments}

The authors would thank the anonymous referees for their constructive remarks that helped to improve the clarity and the completeness of this paper. This work is supported by Young Doctor Foundation of Chongqing Technology and Business University of China (1152003).

\section{References}

[1] D. Ju-Long, "Control problem of grey systems," Systems \& Control Letters, vol. 1, no. 5, pp. 288-294, 1982.

[2] A. M. Andrew, "Why the world is grey," Grey Systems, vol. 2, pp. 112-1116, 2011.

[3] S. F. Liu and Y. Lin, Grey Systems Theory and Applications, Springer, Berlin, Germany, 2010.

[4] S. F. Liu, J. Forrest, and Y. J. Yang, "A brief introduction to grey systems theory," Grey Systems, vol. 2, pp. 89-104, 2012.

[5] T. X. Yao, J. Forrest, and Z. W. Gong, "Generalized discrete GM, (1, 1) model," Grey Systems, vol. 1, pp. 4-12, 2012.

[6] B. Zeng, "Research on electricity demand forecasting based on improved grey prediction model," Journal of Chongqing Normal University (Nature Science), vol. 29, no. 6, pp. 99-104, 2012.

[7] J. Cui and B. Zeng, "Study on parameters characteristics of NGM, (1, 1, k) prediction model with multiplication transformation," Grey Systems, vol. 1, pp. 24-235, 2012.

[8] E. Kayacan, B. Ulutas, and O. Kaynak, "Grey system theorybased models in time series prediction," Expert Systems with Applications, vol. 37, no. 2, pp. 1784-1789, 2010.

[9] G. J. Liu, H. F. Ke, and J. W. Liu, "Equipment development cost and high precision of prediction based on the grey Verhulst optimization model," Military Operations Research and Systems Engineering, vol. 25, pp. 52-56, 2011.

[10] X. Y. Xue and D. S. Fang, "Grey verhulst model for prediction of subsidence of building with soft soil foundation," Underground Space, vol. 21, pp. 515-518, 2001.

[11] D. H. Li, "Verhulst model to predicate ground displacement and deformation," Coal Science and Technology, vol. 32, pp. 58-59, 2004.

[12] Y. C. Liu, "An isochronous grey Verhulst $\operatorname{GM}(1,1)$ model for certain high building subsidence course," The Chinese Journal of Geological Hazard and Control, vol. 17, pp. 61-63, 2006.

[13] Z. X. Wang, Y. G. Dang, and C. G. Shen, "Improvement on Derivative Item in Grey Verhulst Model," Statistics \& Information Forum, vol. 6, pp. 19-22, 2010.

[14] F.-J. Wang, T.-Q. Li, and C.-Z. Yu, "Grey verhulst predictive model of road traffic accidents," Journal of Traffic and Transportation Engineering, vol. 6, no. 1, pp. 122-126, 2006.

[15] F. S. Zhang, F. Liu, and W. B. Zhao, "Application of grey Verhulst model in middle and long term load forecasting," Power System Technology, no. 27, pp. 37-40, 2003.

[16] C. G. Shen, W. M. Chen, and L. L. Pei, "Optimization of the initial conditions in unbiased grey verhulst model," Statistics \& Information Forum, vol. 5, pp. 3-6, 2011.

[17] X. He and W. Z. Wang, "Optimization of grey verhulst model and its application," Journal of Chongqing Institute of Technology (Natural Science Edition), vol. 14, pp. 173-175, 2012.

[18] J. Cui, S. F. Liu, B. Zeng, and M. X. Nai, "Parameters characteristics of grey Verhulst prediction model under multiple transformation," Control and Decision, vol. 28, pp. 605-608, 2013.
[19] Z.-X. Wang, Y.-G. Dang, and S.-F. Liu, "Unbiased grey Verhulst model and its application," System Engineering Theory and Practice, vol. 29, no. 10, pp. 138-144, 2009.

[20] D. L. Yang, S. F. Liu, and B. Zeng, "Verhulst model of interval grey number based on kernel and information field," Control and Decision, vol. 28, pp. 264-268, 2013.

[21] B. Zeng, G. Chen, and S. F. Liu, "A novel interval grey prediction model considering uncertain information," Journal of the Franklin Institute, no. 350, pp. 3400-3416, 2013.

[22] B. Zeng and S. F. Liu, "Calculation for Kernel of interval grey number based on barycenter approach," Transactions of Nanjing University of Aeronautics \& Astronautics, vol. 30, pp. 216-220, 2013. 


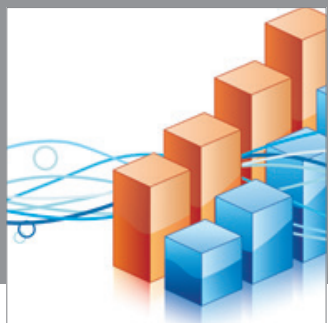

Advances in

Operations Research

mansans

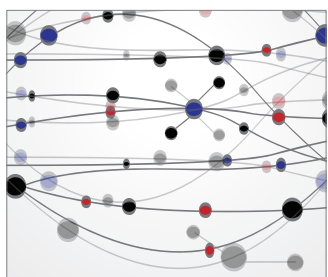

The Scientific World Journal
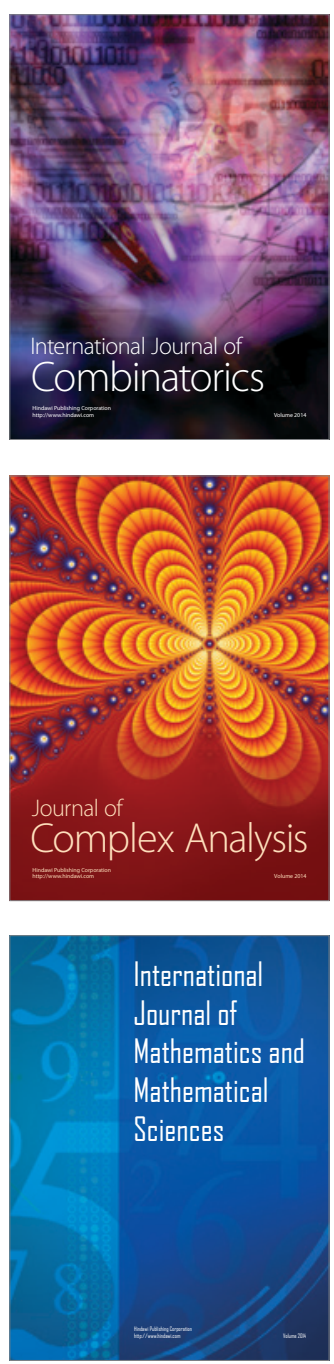
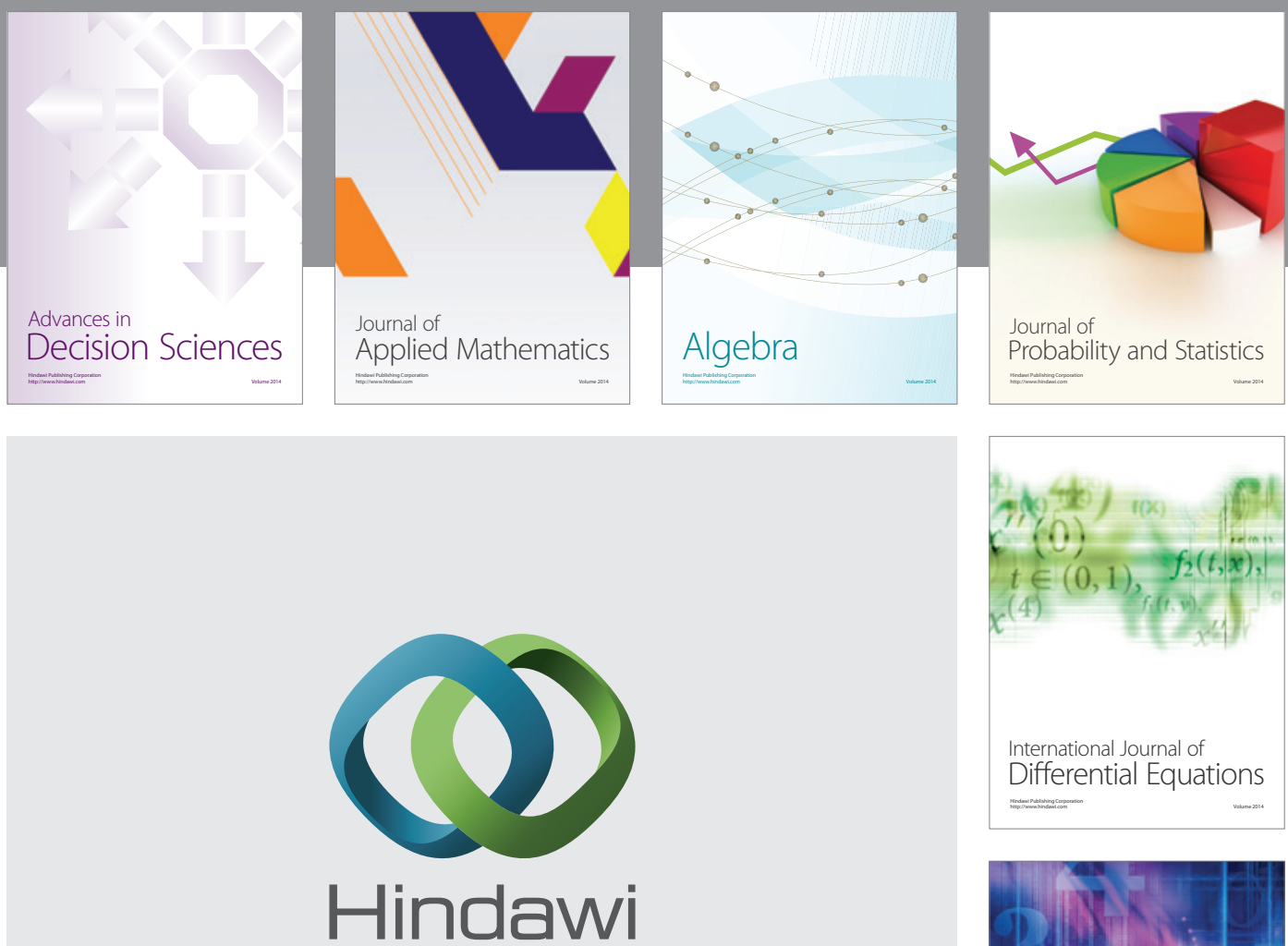

Submit your manuscripts at http://www.hindawi.com
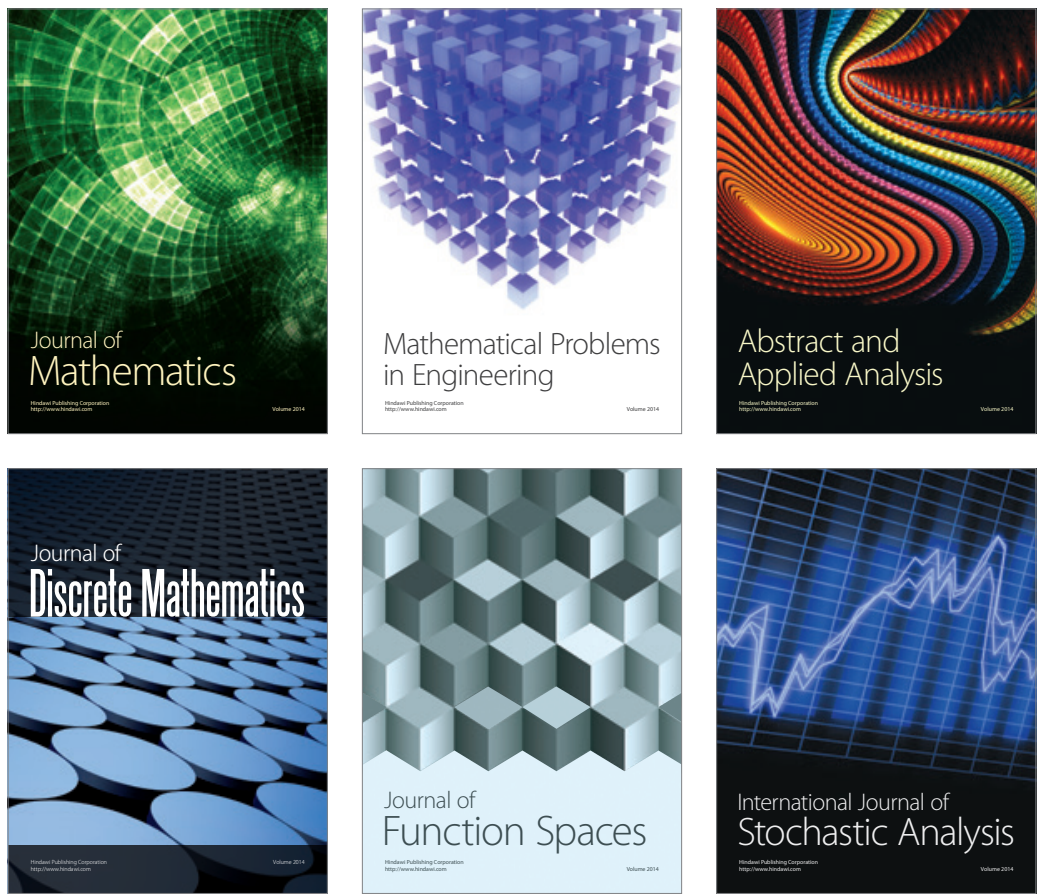

Journal of

Function Spaces

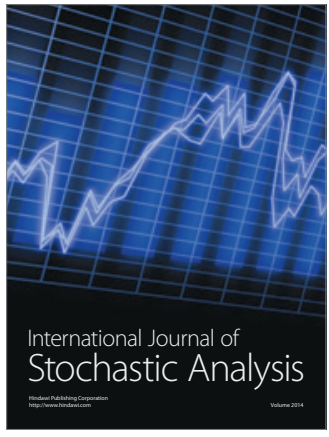

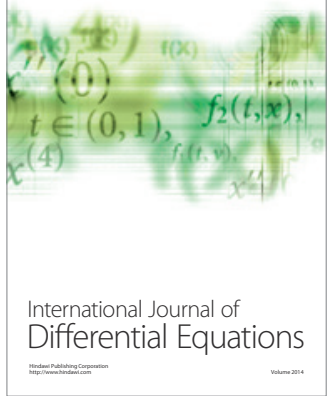
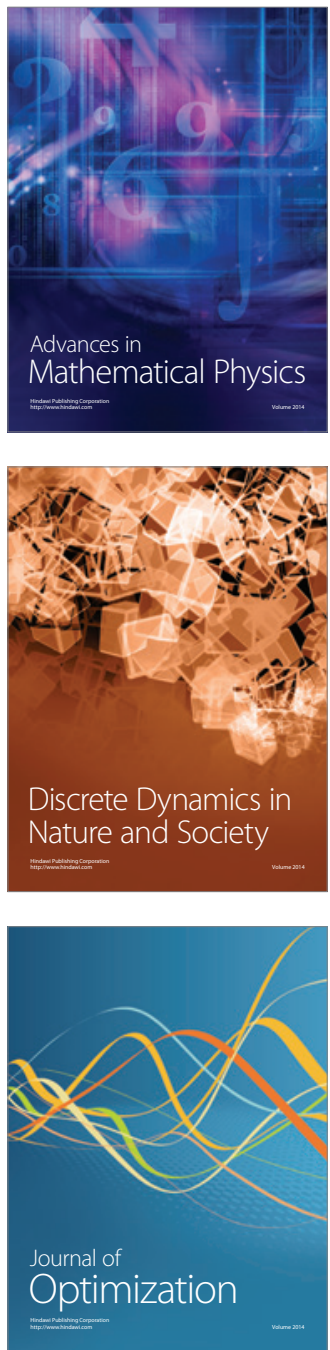